\title{
Directrices del acuerdo ALBA en la Empresa Grannacional en el contexto de ALBA como herramienta promotora del Efecto Multiplicador
}

\author{
Guidelines of the ALBA agreement in the GranNational Company in the context \\ of ALBA as a promoter of the Multiplier Effect
}

Dani José Villalobos Soto'

\section{Resumen}

Tna de las formas de Integración en América Latina y el Caribe ha sido la manera de cooperar que se imprime en estos acuerdos que históricamente ha desarrollado la República Bolivariana de Venezuela y la de Nicaragua en el marco de los acuerdos energéticos, desde la premisa de generar espacios de beneficios para ambas naciones, y de esta manera alcanzar el uso de los grande propósitos que todo país anhela, tales como crecimiento económico, industrialización y economía diversificada de una forma justa y sostenida. La construcción de una empresa Grannancional ha sido el gran desafío que sostiene el acuerdo, cuyo objetivo que se presenta en esta investigación consiste en coordinar directrices del acuerdo ALBA en la empresa Grannacional entre la República Bolivariana de Venezuela y la de Nicaragua en el contexto de ALBA como herramienta promotora del efecto multiplicador en distintos sectores de producción. Esta investigación de tipo documental-descriptivo, el diseño bibliográfico y las fases utilizadas fueron descriptivo, analítico, interpretativo y constructivo.

Se concluye que las asimetrías económicas han constituido el desafío más complejo de América Latina, pero la integración es una estrategia que a través del comercio con las consiguientes ventajas comparativas y competitivas ha permitido incrementar el crecimiento económico.

Palabras Clave: Efecto multiplicador; crecimiento económico; PETROALBA, ALBA; ventaja comparativa; ventaja competitiva; asimetrías económicas.

\section{Summary}

One of the Integration forms in Latin America and in the Caribbean has been the way of cooperating that is printed in these agreements that the Bolivarian Republic of Venezuela and the Nicaraguan Republic have historically developed within the framework of the energetic agreements, from the premise of generating spaces of benefits for both nations, and consequently reach the use of the great purposes that every country desires, such as economic growth, industrialization and diversified economy in a fair and sustained manner. The construction of a GranNational company has been the great challenge that sustains the agreement, the objective that is presented in this research is to coordinate guidelines of the ALBA agreement in the GranNacional company between the Bolivarian Republic of Venezuela and the one of Nicaragua in the context of ALBA as a promoter of the multiplier effect in different production sectors. This documentary-descriptive research, the bibliographic design and the phases used were descriptive, analytical, interpretative and constructive.

\footnotetext{
$\overline{1}$ Magíster en Integración Regional. Profesor Investigador de la Universidad Simón Bolívar. Correo: dvillalobos@usb.ve
} 
It is concluded that economic asymmetries have constituted the most complex challenge in Latin America, but integration through trade is a strategy with the consequent comparative and competitive advantages that has allowed to increase economic growth.

Key Words: multiplier effect; economic growth; PETROALB A; ALBA; comparative advantaje; competitive advantaje; economic asymmetries.

\section{Introducción}

América Latina, se ha destacado por propiciar la cooperación y el comercio en el marco de la integración; desde la década de 1960 son varias las iniciativas que se han organizado en la región para incentivar los esquemas integracionistas $y$ el comercio intrarregional, aun cuando el mayor desafío se concentra en las asimetrías económicas, monoproducción y dependencia. En dicho contexto por iniciativa del ex-presidente venezolano, Hugo Chávez, se creó el acuerdo Alternativa Bolivariana para las Américas (ALBA), en el cual se incluyen varios aspectos de interés obviamente el comercio y también la educación, cultura y telecomunicaciones; en la concepción de un mecanismo favorecedor de las ventajas cooperativas que permitan atenuar las asimetrías existentes entre los países en vías de industrialización.

Para efectos de mayor comprensión científica y académica en el artículo, se describen las funciones de la Empresa Grannacional como organismo que propicia las actividades de PETROALBA empresa cuya objetivo consiste en la comercialización y distribución de petróleo y productos derivados de Venezuela hacia los países que presentan deficiencias en la producción de hidrocarburos y combustibles, ya que no los disponen como recurso natural.

En este sentido, se exponen como premisa de investigación el Tratado como representación de contrato internacional, ALBA y la Empresa Grannacional como mecanismo de integración y la incidencia del Efecto Multiplicador ya que es la técnica que permite propiciar y crear empleos directos e indirectos lo cual conduce a mayor incremento de la producción, ingresos, utilidades de la empresa así como mejora el clima general de la economía y la calidad de vida.
La concepción general del Acuerdo ALBA, tiene por naturaleza apoyarse en el enunciado de la ventaja comparativa y competitiva en la región del Caribe; Venezuela destaca como principal productor y proveedor de petróleo y derivados; sin embargo, presenta debilidades en la producción agrícola y ganadera; por otra parte Nicaragua se destaca en la producción agropecuaria y presenta significativas debilidades en la distribución de combustibles, fertilizantes y otros derivados del petróleo que requieren para la producción agrícola, por ello la premisa que se explica y argumenta es como aplicar las ventajas comparativas y competitivas entre los dos países a partir de la creación de las Empresas Grannacionales y Efecto multiplicador como incentivo del empleo y la producción.

\section{Tratado energético de ALBA entre la República Bolivariana de Venezuela y la República de Nicaragua}

Los vínculos que se establecen entre Estados, ya sean de carácter económico o jurídico y político, implican la regulación de normas, en el sentido que requiere un sistema de principios para funcionar apropiadamente, esto significa el conjunto de principios que según sea el caso, va a regir dicho vínculo; América Latina es una región proclive a la aprobación de acuerdos de cooperación cuyos gobernantes han tenido especial interés en propiciar mecanismos de integración; la posición de los países de la región se ha caracterizado por la conciliación e incentivos a la integración.

En este sentido se plantea como premisas del ensayo la figura del Tratado como contrato internacional que propicia los mecanismos de integración ALBA, la Empresa Grannacional es un instrumento proveedora de bienes, servicios, empleos y utilidades que se apoya en el Efecto Multiplicador para tal fin y los vínculos que se establecen con el comercio, los cuales se contemplan en la esencia misma del acuerdo.

Todo acuerdo debe regirse por normas, que se expresan en los principios del Derecho Internacional Público, se incluye la definición de Tratado por Salazar (2005) de acuerdo a lo consagrado en la Convención de Viena sobre el derecho de los tratados de 1969 se entiende por tratado, "un acuerdo internacional celebrado por escrito entre Estados y regido por el Derecho Internacional, ya conste en un instrumento único o en dos o mas instrumen- 
tos conexos cualquier a que sea su denominación particular" (p.35).

Por consiguiente, el tratado es el documento, que le concede carácter legal, jurídico y formal a un acuerdo, predomina la normativa de la Convención de Ginebra de 1969 que lo define como un acuerdo celebrado entre Estados por escrito y acorde al derecho internacional ya sea en varios documentos o en uno, de modo que se concibe como un contrato que compromete a los Estados Partes, y consta de varias etapas tales como ratificación, aceptación, aprobación y adhesión.

En términos jurídicos, una vez que los Estados celebran el Tratado, el procedimiento de mayor relevancia lo constituye la ratificación ya que consisten en la aceptación por los países firmantes del acuerdo y la disposición y compromiso que demuestran para hacerlo cumplir, a que el mismo concede derechos y deberes, el acto de ratificar obliga a los países a cumplir con los principios que se describen en los tratados, Universidad Católica de La Plata (2009) citado por Salazar aporta la siguiente explicación:

Se entiende por "plenos poderes" un documento que emana de la autoridad competente de un Estado y por el que se designa a una o varias personas para representar al Estado en la negociación, la adopción o la autenticación del texto de un tratado, para expresar el consentimiento del Estado en obligarse por un tratado, o para ejecutar cualquier otro acto con respecto a un tratado (Ob Cit.).

Como ya se ha descrito anteriormente, el Tratado obliga a las partes, a cumplir el contenido del acuerdo, los plenos poderes es la concesión y autoridad que se le concede al representante de un Estado, cuando el tema del Tratado es de índole económica se considera que es una modalidad de contrato internacional en la cual las partes no solamente se comprometen, sino que adquieren deberes cuyo objetivo es el estricto cumplimiento y derechos que están en capacidad de exigir según sea el contexto.

Venezuela constituye en la región el país proveedor de petróleo y derivados más relevante de América Latina, con una posición geográfica privilegiada; con fachada frontal al Mar Caribe y fácil acceso a las Antillas Mayores, Menores y
Centroamérica ya sea por comunicación marítima o aérea; en contraposición a tal descripción los países caribeños presentan un significativo potencial turístico y pesquero pero adolecen de combustibles y energía, como también algunos países de Centroamérica cuya sistema económico lo constituye la agricultura, pero presentan igual escasez de combustibles.

Cuando los países presentan limitaciones inherentes a la disponibilidad de recursos naturales, ya sea caudales hidráulicos, petróleo, gas natural, superficie de tierra cultivable, o recursos mineros, las expectativas y posibilidades de desarrollo y crecimiento económico están limitadas; la respuesta a tal carencia se encuentra en la cooperación, integración, el establecimiento de vínculos comerciales justos y equitativos o la implementación de políticas eficientes para contrarrestar los efectos de la escasez de recursos.

\section{Definición y Funciones de Empresa y de Empresa Grannacional en el contexto de PETROALBA}

En la dinámica del Comercio Internacional, el desempeño de las empresas constituyen un factor fundamental para el funcionamiento del mismo, ya que significan unidades de producción y prestación de servicios que dinamizan el intercambio comercial, el concepto se asocia frecuentemente con el bien privado y el sistema capitalista; sin embargo, la historia ha demostrado que el Estado también incursiona como empresario, toma iniciativas gerenciales e incluso puede erigirse como un significativo núcleo generador de empleo y producción, así presenta coincidencia con el efecto multiplicador, se destaca la definición de Empresa que aporta Gil ( 2007) citando a Zerrilli :

La empresa es la suma de diferentes componentes; un objeto común, objeto de personas y medios, un esfuerzo combinado y un sistema de dependencias y relaciones que aseguren la coordinación. Así define la empresa como un conjunto de medios humanos y materiales que se disponen para conseguir la finalidad según un esquema determinado de relaciones y dependencias entre los diferentes elementos que la componen (p. 30).

De acuerdo a la cita anterior, la empresa es una organización en la cual convergen recursos huma- 
nos, materiales tangibles e intangibles esencialmente una unidad de producción, o prestaciones de servicios cuya naturaleza en ocasiones es impalpable, cuando dicha afirmación se extrapola a un contexto más amplio y macro como lo es el Estado, significa el funcionamiento de instituciones cuyas normas y parámetros se establecen a través de Tratados o instrumentos jurídicos como documentos escritos que comprometen a los Estados Partes, ya que se consideran los mismos un contrato y como tal concede una serie de derechos y deberes cuya naturaleza está regida por la ética. se destaca una segunda acepción por Samper (2013):

Por empresa se entiende la organización de elementos personales y materiales dedicada a producir y transformar bienes y a prestar servicios: El calificativo de pública la diferencia de la privada tan solo por el carácter público cualidad que no afecta de modo alguno a su régimen de tráfico. (p.54)

Visto de esta forma, una empresa es una unidad de producción que transforma insumos y materias primas, así como presta servicios para satisfacer la demanda de los consumidores, cuando dicha iniciativa parte del Estado, los recursos provienen del erario público y el acuerdo, organización y estrategias se establecen a través del marco jurídico que implican los tratados con carácter de contratos; si bien en ocasiones se ha cuestionado la participación del Estado como ente empresarial, también ha realizado importantes gestiones especialmente para incentivar la producción en zonas económicamente deprimidas; lo cual conduce al autor a plantear la premisa ¿Cómo se establece un proceso de implementación de Empresa Grannnacional como incentivo del empleo y aplicación al Efecto Multiplicador en el contexto del acuerdo ALBA?

El acuerdo es la voluntad manifiesta de los Estados, para establecer normas en una materia común, si se compara con las características del Acuerdo de Cooperación Energética PETROALBA a fin de sustentar con bases el objeto del mismo, explica en los siguientes términos: "Un acuerdo de cooperación es aquel donde se resuelven las diferencias existente entre las partes contratantes, además se deben recoger aspectos de deficiencias de cada una de las partes a fin de evitar ambigüedades durante su desarrollo." (Disponible: http:/ alba-tcp.org).
La concepción de cooperación ya implica que existe la disposición consciente y formal de resolver diferencias por medios legales y satisfactorios para las partes contratantes, por lo cual la naturaleza y dimensión de los acuerdos de cooperación tiene connotación comercial, territorial o de seguridad y defensa; para abogar temas desde el libre comercio hasta la prevención y control de actividades como el narcotráfico, se evidencia en el siguiente extracto del Tratado: "Resolver las asimetrías en el acceso a los recursos energéticos, por la vía de un nuevo esquema de intercambio favorable, equitativo y justo entre los países de la región caribeña". (I bid.)

En este sentido, aplicar estrategias para resolver las asimetrías se considera el desafío mas complejo en las etapas de integración; equipara profundas diferencias en los modos de producción, tecnología, ventajas comparativas y competitivas no es tarea sencilla; sin embargo, si se aplica una óptima y eficiente red de programas, acuerdos y estrategias públicas, patrocinadas por los Estados, existen amplias oportunidades de insertar progresivamente a las zonas menos productivas o con índice tecnológicos muy bajos, a la producción general de un país.

La naturaleza intrínseca de la cooperación, se asocia con la disposición de buena voluntad a apoyar lealmente sin contraprestación alguna;empero la dinámica propia del comercio actual ha contribuido a modificar dicha valoración en el sentido que en el contexto de ALBA la cooperación implica un intercambio razonable y factible de bienes y servicios como estrategias y mecanismo para incentivar el comercio intrarregional; una de las razones que facilita un proceso de industrialización estancado se refiere a la aplicación de directrices ajenas a la realidad latinoamericana, cuando se aspira a sustituir esta medida por un intercambio razonable los resultados favorecen más nuestro contexto.

Para Venezuela la importancia geopolítica en la zona de influencia en los programas de cooperación es vital por múltiples razones. Por seguridad y defensa del territorio nacional, porque es ruta segura de más del 80\% de las exportaciones venezolanas, porque una gran parte de la población habita sobre los $2.256 \mathrm{~km}$ de costa caribeña, porque representa un potencial mercado para productos venezolanos así como por sólidos e históricos lazos de hermandad con países vecinos. Además de esto es preciso hacer mención sobre los aspectos a considerar en un acuerdo de cooperación los cuales para el autor son los siguientes: 
La cooperación tiene dos niveles de interpretación y aplicación: cuando se realiza por razones humanitarias y cuando es el producto del amplio consenso entre las partes que presentan problemas, metas e intereses en común como lo es la región caribeña en la cual el desarrollo del comercio e integración constituyen factores primordiales para toda la región; por lo tanto, se interpreta como el segundo caso.Esto significa que la cooperación es una dinámica interacción de ofertas y demandas en la región que a su vez se compaginan con la ventaja comparativa y competitiva.

En los objetivos antes mencionados dan características específicas para identificar los acuerdos de cooperación, en este caso tiene vinculación con la iniciativa energética PETROALBA por medio de este se observa la participación de diversas actividades tales como son: la producción, el servicio, la distribución, así como también la participación de cada país miembro para cumplir con los objetivos propuestos, se destaca la explicación exhaustiva de PETROALBA como acuerdo regional que a continuación describe:

Alternativa Bolivariana para los pueblos de América se define como una propuesta realizada por la República Bolivariana de Venezuela y Cuba orientada a los países de America latina y el Caribe, la creación de este mecanismo responde a aliviar las asimetrías existentes entre los países que lo conforman, además el ALBA se apoya en principios de solidaridad, complementariedad y cooperativismo.

\section{Actividad Empresarial y Efecto Multiplicador}

De acuerdo a las explicaciones anteriores, la creación de empresas implica un conjunto de beneficios tanto para trabajadores como patronos; son fuentes de empleo, estabilidad, crecimiento económico, ingresos, renta y prestación de servicios, sin embargo no siempre las condiciones se erigen de manera idónea, existen casos en los cuales por razones estructurales del funcionamiento de la economía no se evidencian condiciones para mantener y crear un sector empresarial, o es muy pequeño y poco representativo, razón por la cual el nivel de ganancia y rentabilidad no es realmente importante, factor muy desfavorecedor si se considera que todos los países requieren un sector empresarial consolidado para propiciar y mantener la producción por ello es necesario aplicar otras estrategias como lo es el Efecto Multiplicador que explica Ávila y Lugo (2004) en los siguientes términos :

Es la razón del aumento del empleo global, respecto al aumento de la inversión... indica el número de personas que se añadirán al empleo original, por cada uno que sea directamente empleado. El empleo indirecto o secundario es el que se origina en las industrias y empresas a consecuencia del empleo primario en las mismas, debido a las inversiones que se efectúan (p. 95).

De modo que el propósito del Efecto Multiplicador, se orienta a incrementar el empleo y ello se logra creando estructuras empresariales que propicien la producción, los ingresos y actividades laborales directas, indirectas relacionadas a la empresa; ello implica la participación de varias sectores en el proceso de producción; desde la refinación del insumo hasta la distribución del mismo por ello se afirma que el sector construcción y servicios constituye uno de los sectores que facilita mas rápidamente la creación de empleos de actividades que se requiere, además que se considera apropiado en regiones con escasa tecnología o producción muy baja, se incluye otra acepción por Pearce (1999) :

La relación de un cambio en la renta respecto al cambio inicial en el gasto que lo produjo...es la variación en la renta nacional y en las inyecciones dará como resultado un incremento en el flujo anual de la renta nacional del doble de esta magnitud. El aumento en la inversión Provoca un incremento de la renta que a su vez se gastará parcialmente en otros bienes y servicios (p. 273).

En otras palabras, la creación de nuevas fuentes de empleo requiere inversión en estructuras, equipos y tecnología que propicien la contratación de trabajadores para utilizar dichas maquinarias, a su vez implica inversión y gasto que se retribuye cuando la producción a mediano plazo es suficiente para recuperar la inversión, si a la premisa inicial se agrega el hecho que el comercio entre Venezuela y Nicaragua es favorecedor en términos de ventajas competitivas ya que el primero requiere productos agrícolas y alimenticios que no produce en su totalidad mientras que Nicaragua requiere combustibles y derivados para transportar dichos productos. El Efecto Multiplicador presenta varias ventajas tales 
como la expansión de la clase empresarial cuya ocupación sea el sector agroindustrial.

\section{Opciones de las Empresas Grannacionales en los Sectores Productivos}

Las empresas Grannacionales en el contexto de ALBA, significan una extensa y compleja red de interacciones inherentes a distintas categorías de actividad económica, educativa y comercial alineadas con los principios del tratado, los acuerdos se contemplan entre dos o más países cuyo propósito esencial consiste en promover la producción, divulgar nuevos mecanismos de producción, lo cual incluye comercio justo y reduce las asimetrías en condiciones equitativas.

La Alianza político estratégica que significa ALBA se dirige a propiciar formaciones económico-sociales, cuya esencia y naturaleza sea el desarrollo equitativo que reduzca significativamente las asimetrías y favorezca la igualdad para la inserción eficiente en el mundo actual, en un contexto mucho más humano en el cual la política cumpla la función de erigirse como mecanismo y estrategia para mejorar las condiciones y calidad de vida de los ciudadanos.

Cuando las condiciones en los países no son equitativas, ni se propicia la inclusión social va a generar a mediano y largo plazo desigualdad y asimetría que se traduce en pobreza en un amplio margen de la población, que no alcanza la satisfacción de necesidades, mínimas ni de acceso a los servicios públicos y evidencia asimétrica, en ocasiones los planes amplios, ambiciosos y muy extensos han demostrado que no logran los objetivos pre-determinados, por el contrario las estrategias más modestas y sencillas logran obtener resultados más eficaces, en este sentido la sub-división del ALBA en varios sub-rubros económicos propicia mayor crecimiento.

Sin embargo, el problema de las asimetrías no es tarea sencilla de resolver; existe la contradicción entre la aspiración de varios países de lograr la industrialización completamente proceso que implica una gran inversión de tiempo y dinero e incluso puede extenderse por décadas, en la actualidad se está cuestionando en el sentido si es una propuesta factible con las características de cada país y la opción de ser competitivo con los recursos y la producción disponible, sin que ello implique una extensa industrialización, además de buscar el apoyo de las alianzas integracionistas, se considera oportuno incluir la explicación de Altmann (2011) :

El ALBA diseña e implementa programas sociales en educación, salud, empleo, alimentación y distribución de canasta básica. Programas sociales financiados por el Estado, dirigidos a beneficiar las amplias mayorías populares y a los sectores vulnerables y a partir de la participación popular, dando así respuesta a la inmensa deuda social acumulada no solamente durante el largo interregno neoliberal sino también por los siglos de explotación colonial y neocolonial (p.186).

Por consiguiente, se ha concebido como un mecanismo para dar respuesta a distintos sectores de una forma distinta a la estrategia anterior, tan sesgada y subordinada a los intereses de las grandes potencias, el acuerdo macro contempla varias clasificaciones que incluyen comercio, telecomunicaciones, alimentación, turismo, industria, minería, y transporte.

Con el propósito de coordinar e implementar actividades en distintos sectores en el seno del ALBA se creó el Banco-ALBA para aplicar mecanismos eficientes y cónsonos a los programas de desarrollo cuyo propósito consiste en la eliminación de asimetrías económicas, cada país participante entrega un aporte con el propósito de estimular la renta nacional considerando las peculariedades de la región y las diferencias que existen entre los niveles de industria.

Ciertamente es un enfoque distinto a la cooperación que la competitividad. El Banco ALBA no es una institución financiera para que las naciones compitan con el fin de establecer cual reúne las opciones que lo hacen apto para un crédito, se refiere a un estudio exhaustivo para determinar las opciones de desarrollo desde los recursos disponibles, en el contexto de países que no se caracterizan por altos ingresos creando vínculos comerciales flexibles mas favorecedores a la región latinoamericana y caribeña, en el cual prevalecen la solidaridad y cooperación, el estudio para optar a los créditos se orienta en evaluar las posibilidades que tienen los países con menos recursos y como crear una estrategia que les permita producir sin crear un endeudamiento excesivo. 
La versatilidad del tratado, funciona para abordar distintos sectores como ALBA-Comercio, que se evidencian en el Tratado de Comercio de los Pueblos TCP como una estrategia cuyo propósito consiste en incentivar, propiciar y estimular el comercio interregional, la ventaja comparativa y competitiva en contraposición a las normas estrictas del neoliberalismo, es oportuno incluir la acotación de Sabino (2011) acerca de neoliberalismo:

El neoliberalismo acepta que el Estado puede tener un papel definido en la economía, especialmente en cuanto a la conservación de los equilibrios macroeconómicos y el desarrollo de una limitada política social, pero comparte con los liberales de los siglos XVIII y XIX su misma convicción sobre las virtudes de una economía de libre mercado y las ventajas de un ordenamiento que estimule el desarrollo libre de la persona humana en todos los campos de actividad (Disponible: eumed.net).

De allí pues, que como doctrina económica le concede una mayor prevalencia a la competencia de la oferta y la demanda en el mercado que cuestiona la intervención del Estado, se excede en la flexibilidad, de tal manera, como doctrina económica, le concede mayor relevancia a la interacción de las empresas como sector privado, que a la participación del Estado en la economía, si a ello se suma que en los países latinoamericanos se evidencia una marcada tendencia a observar procesos de industrialización incompletos, e injusta distribución de la riqueza así como un sector empresarial que en ocasiones es de menor dimensión que la propia capacidad del Estado, se infiere que no es la doctrina mas conveniente para dicho contexto.

Por lo tanto el acuerdo ALBA, propicia condiciones comerciales más igualitarias, justas y humanas de esta manera, una de las propuestas consiste en adquirir la producción agrícola de Bolivia, especialmente de cereales como la quinoa y semillas de chía para incentivar las exportaciones de dicho país y mejorar el sistema económico, ya que la integración no es sólo un acuerdo de intención cooperativa, también se trata de expandir el comercio intrarregional aprovechando las ventajas comparativas y competitivas de cada país participante, en términos equitativos que realmente contribuyan a reducir las asimetrías.
La metodología de ALBA, implica un procedimiento innovador que propicia los cambios en la política, burocracia y grupos de interés a través de los programas sociales. Se establece una amplia acción de posibilidades que con los procedimientos de inspección, auditoría y evaluación mejoran la política administrativa y la planificación estratégica, el acuerdo ha sido concebido con una profunda complejidad que atañe a todos los temas de interés para la ciudadanía y los respectivos gobiernos.

En ocasiones la celebración de acuerdos integracionistas, se concentran exclusivamente en las razones comerciales o territoriales si es el caso y excluyen otros aspectos que igualmente son relevantes en dicho sistema tales como la cultura, educación, deportes, transporte y salud que interactúan para incentivar el bienestar en la población y también como efecto multiplicador para crear nuevas fuentes de empleo y producción; ALBA es un acuerdo tan completo y ambicioso que prevé la transferencia de recursos humanos para cumplir vacantes que requieren de trabajadores y profesionales en distintas especialidades.

En la clasificación para establecer normativas en cada sector de interés, se demuestra específicamente en el propio contenido del tratado por ALBA (2008): "Artículo 3: Los países intercambiarán paquetes tecnológicos integrales desarrollados en sus países por las partes, en áreas de interés común, que serán facilitados para su utilización y aprovechamiento, basados en principios de mutuo beneficio". (Disponible: http:/alba-tcp.org).

Es por eso, que, en la dimensión y magnitud del artículo pre-citado, se evidencia las intererrelaciones que establece el acuerdo entre distintas categorías como industria, alimentación, transporte, minería y salud, se refiere a la incentivación y expansión del desarrollo endógeno local y regional para lograr la autosuficiencia y eliminar la dependencia de las exportaciones.

\section{Conclusiones}

Una vez que se ha descrito exhaustivamente el funcionamiento de la Empresa Grannacional y el Efecto Multiplicador el autor determina las siguientes conclusiones:

Las asimetrías económicas, constituyen el desafío mas complejo que experimenta la región 
latinoamericana; sin embargo, la integración se erige como una significativa estrategia que a través del comercio y la aplicación eficiente de las ventajas comparativas y competitivas permite incrementar el crecimiento económico.

La Empresa Grannacional se ha concebido, como una extensa red que contribuye a incentivar la producción, empleos y consumo desde una perspectiva regional en concordancia con las necesidades y potencialidades de cada país participante; en términos económicos lo que esencialmente necesita un país para recuperarse es producción lo cual conduce indudablemente crear fuentes de empleo directa o indirectamente, esto es la función principal del Efecto Multiplicador; es un factor que propicia por su propia naturaleza el empleo al incrementarse la cantidad de trabajadores y unidades de producidas se genera mayores.

El mecanismo para incentivar las ventajas comparativas y competitivas entre Venezuela y Nicaragua se concentra en el amplio grado de adaptabilidad al medio y asimetrías latinoamericanas, a través de él se crean una amplia variedad de opciones para cultivar productos autóctonos con el propósito de la refinación y comercialización, permite la explotación de especies desconocidas, así como la exportación de los mismos como frutos exóticos, facilita el desarrollo en otros sectores económicos como manufactura y agro-industria, además que genera empleos bajo el marco legal del acuerdo ALBA.

\section{Lista de referencias}

ALBA, (2008). Acuerdo ALBA -TCP [Documento en Línea] (Disponible: http:/alba-tcp.org).

Altmann, J. (2011). América Latina y el Caribe: ALBA ¿Una Nueva Forma de Integración regional? Editorial Teseo Buenos Aires, Argentina.

Ávila, L. y Lugo, M. (2004). Introducción a la Economía. Editores Plaza y Valdés. México D. F.

Gil, M. (2007). Cómo Crear y Hacer Funcionar una Empresa: Conceptos e Instrumentos. ESIC Editorial. Madrid. $7^{a}$ Edición.

Espinoza, E. (2014). El ALBA-TCP: Integración Bolivariana en Nuestra América [Texto en Línea] (Disponible: http: //WWW. eumed. net).

Pearce, D. (1999). Diccionario Akal de Economía. Ediciones Akal. Madrid, España.

Rodríguez A. (2008). El Banco del Sur y el Banco del ALBA Observatorio de la Economía Latinoamericana $\mathrm{N}^{\circ}$ 93, [Texto en Línea] (Disponible: http: //www. eumed.net).

Sabino, C. (2011). Diccionario De Economía y Finanzas. Editorial Panapo [Texto en Línea] (Reedición) (Disponible: http:// carlossabino. freeservers.com).

Salazar, M. (2005). Fundamentos del Derecho Internacional Público. Editorial El Nacional, Caracas, Venezuela. 